\title{
Simulation and Measurement of HTS Josephson Heterodyne Oscillator
}

\author{
John Cameron Macfarlane, J. Du, R. Taylor, and C. M. Pegrum
}

\begin{abstract}
We report continuing investigations into practical applications of the ac Josephson effect as the basis for a voltage-tunable radio-frequency oscillator. We have previously demonstrated experimentally that useful power levels ( $10 \mathrm{~s}$ of $\mathrm{nW})$ and linewidths of a few $\mathrm{kHz}$ can be achieved in the heterodyne output from a High-Temperature-Superconducting Resistive SQUID (HTS-RSQUID) operating in the frequency range 1-50 MHz. Those results were achieved with 2 -junction R-SQUIDs incorporating current-biased shunt resistors of a few micro-ohms. We have now modified the fabrication procedures, and adjusted the shunt resistors and bias current values so that higher frequencies can be achieved. The Josephson junctions are of step-edge type, rather than the bi-crystal type used in our earlier work. The step-edge technique permits much more flexibility in the geometrical lay-out and utilizes the more cost-effective single-crystal MgO substrates. In the present paper, we report numerical simulations and experimental measurements on these devices in the frequency range up to $2 \mathrm{GHz}$.
\end{abstract}

Index Terms-Heterodyne oscillator, high-temperature-superconducting, Josephson junction.

\section{INTRODUCTION}

$\mathbf{P}$ RACTICAL applications of the ac Josephson effect for the generation of useful radio-frequency oscillator outputs in the $\mathrm{MHz}$ to $\mathrm{GHz}$ range have been difficult to realize, partly because the applied voltages are required to be inconveniently small ( $<1$ microvolt), but primarily because intrinsic junction noise contributes to linewidth broadening of the emitted radiation. Typically, Johnson noise at temperatures of $50 \mathrm{~K}$ in the junction resistance of order $10 \mathrm{ohms}$ can lead to linewidths of $500 \mathrm{MHz}$ or more, which is unacceptable in most applications that might be envisaged.

We shall instead exploit the intrinsic non-linearity of Josephson devices to generate heterodyne signals in the $\mathrm{MHz}$ to $\mathrm{GHz}$ range from two oscillating junctions connected in the resistive-SQUID configuration [1]. This device consists of two Josephson junctions J1, J2 connected in series in an

Manuscript received August 19, 2008. First published June 05, 2009; current version published July 10, 2009. This work was supported in part by the CSIRO, Australia, and Mesaplexx Pty Ltd, Australia.

J. C. Macfarlane is with the Physics Department, University of Strathclyde, Glasgow G4 0NG, UK (e-mail: j.c.macfarlane@ strath.ac.uk).

J. Du is with CSIRO Materials Science and Engineering, Lindfield 2070, Australia.

R. Taylor is with Mesaplexx Pty Ltd, Qld, Australia.

C. M. Pegrum is with FieldSolutions, Glasgow G12 9SD, UK. He is also with the Department of Physics, University of Strathclyde, Glasgow G4 0NG, UK.

Color versions of one or more of the figures in this paper are available online at http://ieeexplore.ieee.org.

Digital Object Identifier 10.1109/TASC.2009.2018803

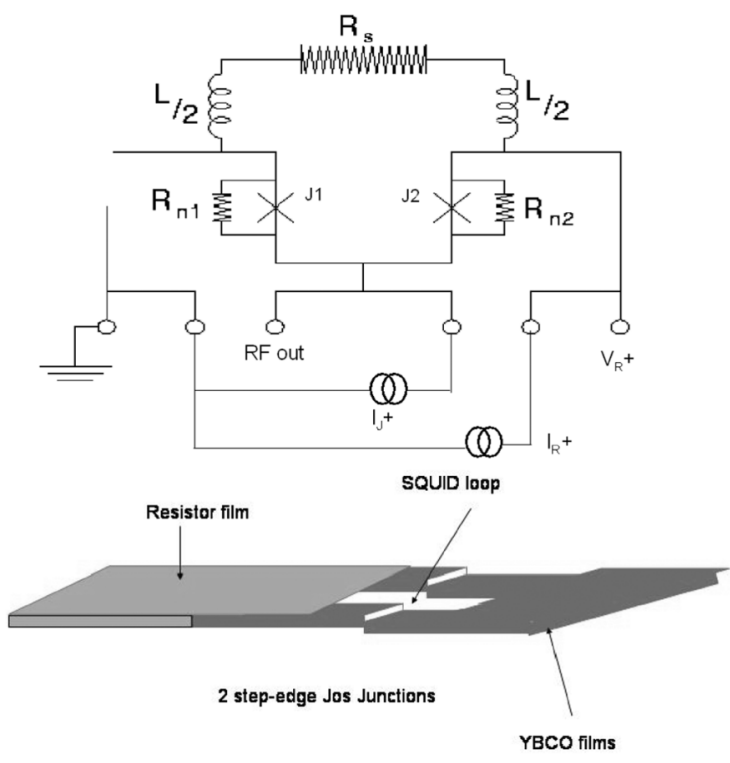

Fig. 1. (Upper), schematic circuit of R-SQUID device; components are explained in the Text; (lower:), sketch of thin-film layout used to produce the R-SQUID.

otherwise superconducting loop containing a small resistor $R_{S}$ (see Fig. 1). A sketch of the thin-film geometry is also shown in Fig. 1.

The resistors $\mathrm{R}_{\mathrm{n} 1}, \mathrm{R}_{\mathrm{n} 2}$ represent the normal resistance of the step-edge junctions, typically $2-10 \mathrm{ohms}$. The inductors $\mathrm{L} / 2$ represent unavoidable inductances in the device structure, and are estimated to be in the range $10-20 \mathrm{pH}$. Currents $\mathrm{Ij}$, Ir are supplied from dc sources, and the heterodyne rf output is extracted via a dc blocking capacitor from the indicated terminal to a co-axial cable. With this arrangement, the separate junctions are voltage-biased and typically oscillate at $10-100 \mathrm{GHz}$, while the heterodyne output can be precisely controlled by adjustment of $\mathrm{I}_{\mathrm{r}}$ in the $100 \mathrm{MHz}$ to $2 \mathrm{GHz}$ range, with an intrinsic linewidth determined by Johnson noise in $\mathrm{R}_{\mathrm{s}}$ :

$$
\Delta \mathrm{f}=4 \pi \mathrm{kTR} / \phi_{0}^{2}
$$

where $\mathrm{k}$ is Boltzmann's constant, and the flux quantum $\phi_{0}=$ $2 \times 10^{-15} \mathrm{~Wb}$.

Before experimental work was started, we carried out numerical simulations of the devices' performance, using JSIM and JSPICE3 circuit analysis software packages, primarily to look at the behavior in the time domain. An example of the output obtained from this modeling is shown in Fig. 2. The step-edge junctions were modeled as Resistively-Shunted-Junctions. More extensive modeling results are presented in greater detail in a companion paper at this Conference [2]. 


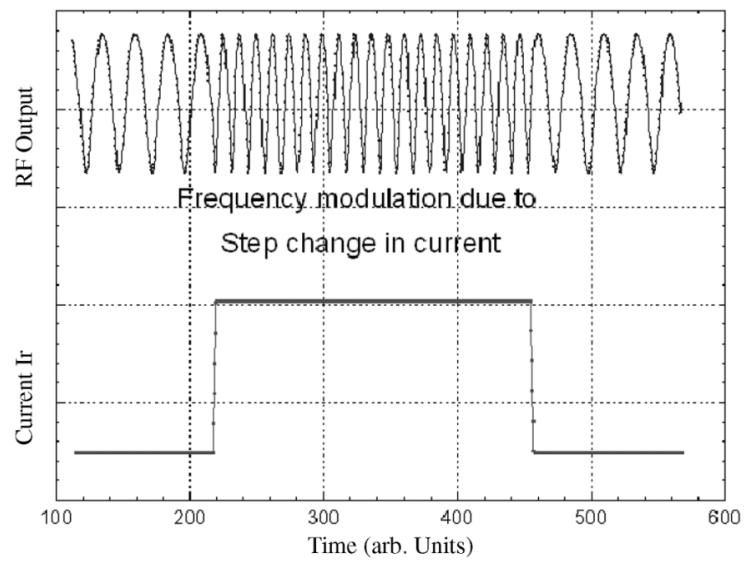

Fig. 2. Example of time-domain simulation of an R-SQUID, obtained by JSIM. The response of the heterodyne frequency to a step change in the current Ir is illustrated.

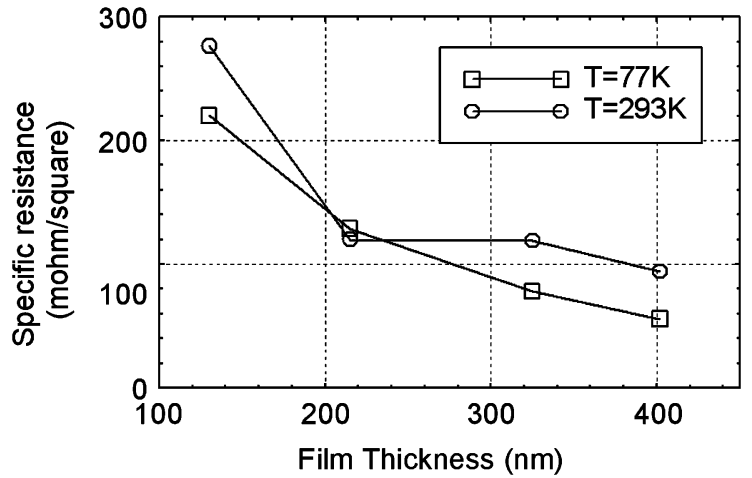

Fig. 3. Measurements of the Au-film specific resistance as a function of thickness, at $77 \mathrm{~K}$ and at room temperature.

\section{EXPERIMENTAL APPROACH}

Earlier work [3] used resistors $R_{S}$ with values around 20 micro-ohms, with which $\Delta \mathrm{f}$ is expected to be of the order 17 $\mathrm{kHz}$ at $\mathrm{T}=20 \mathrm{~K}$. Heterodyne frequencies obtained at that time were in the range $1-50 \mathrm{MHz}$, with bias currents in the range 0-5 mA. In the present phase of this project, the frequency of interest is extended to $\sim 2 \mathrm{GHz}$. To meet this requirement, either (i) the resistor $R_{S}$ has to be increased by about a factor of 100 into the milli-ohm range; or (ii) bias currents $I_{r}$ have to be increased by a similar factor. In practice, we used Au-film resistors of order 1-10 milli-ohm, and bias currents in the range $0.2-1 \mathrm{~mA}$, giving a theoretical range of heterodyne frequencies of $0.2-10 \mathrm{GHz}$.

\section{A. Resistors}

Before the micro-lithographic masks were designed, it was necessary to obtain a realistic value for the specific resistance of our Au films, so that the desired resistor values of 1-10 mohms could be achieved. Four samples of Au film resistors were prepared with different film thicknesses $(100 \mathrm{~nm}-400 \mathrm{~nm})$, and the specific resistance values measured at room temperature and liquid N2 temperature ( $77 \mathrm{~K}$ ) (Fig. 3). To avoid large contact resistances, however, it was essential to deposit in-situ a layer of Au on top of the newly-deposited YBCO [4].

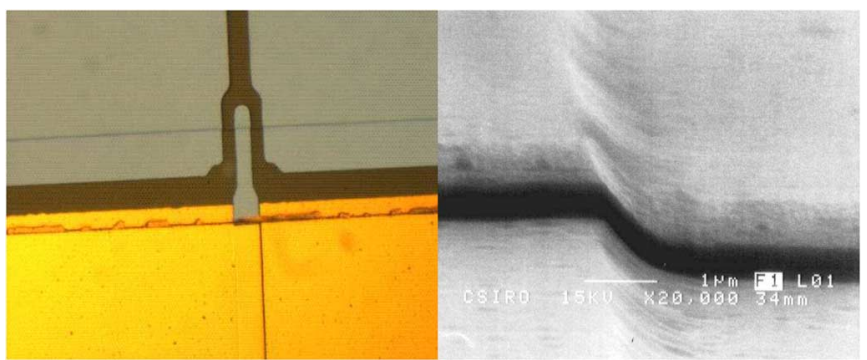

Fig. 4. A photograph of a fabricated device junction area (left) and an SEM micrograph of the YBCO step-edge junction (right). The step, produced on the $\mathrm{MgO}$ substrate by Ar-ion beam etching, is $\sim 400 \mathrm{~nm}$ in height. The magnification is $\times 20,000$.

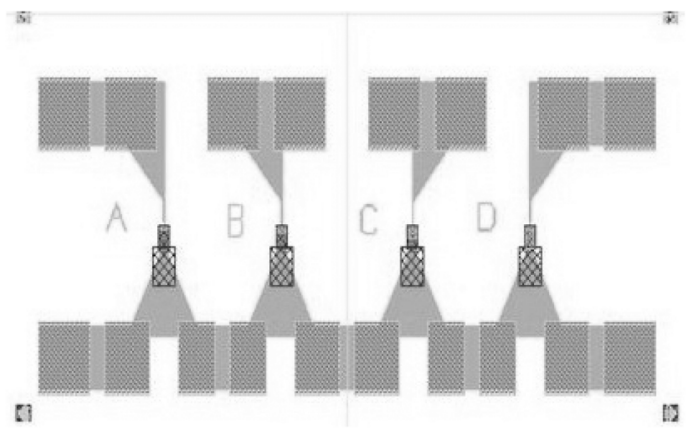

Fig. 5. Sketch of the 4-device layout on a $5 \mathrm{~mm} \times 10 \mathrm{~mm}$ chip. Hatched rectangles represent the $\mathrm{Au}$-film resistors.

\section{B. Junctions}

The devices are based on the established step-edge YBCO junction technology developed at CSIRO [5], [6]. The $\mathrm{MgO}$ substrates were first etched by Ar-ion beam to produce steps of $\sim 400 \mathrm{~nm}$ high in the desired positions. The $200-220 \mathrm{~nm}$ thick YBCO films, and $50 \mathrm{~nm}$ in-situ Au films, were then deposited by Theva $\mathrm{GmbH}$ [7]. The $10 \mathrm{~mm} \times 10 \mathrm{~mm}$ substrates were then returned to CSIRO, where eight devices of variable designs were fabricated on each one. A photograph of a fabricated device junction area and an SEM micrograph of a typical step-edge junction are shown in Fig. 4. The devices are highly reproducible, on-chip and from chip to chip, as evidenced by critical-current and resistor measurements [5], [6].

The finished substrate was diced into two $5 \mathrm{~mm} \times 10 \mathrm{~mm}$ chips (Fig. 5) for packaging and measurements. The simultaneous fabrication of multiple devices enabled the variability of some important device parameters, such as $R_{s}, I_{c}$ and $R_{n}$, to be assessed at an early stage of device development.

\section{EXPERIMENTAL RESULTS AND DISCUSSION}

Initial tests were done while cooling the device in the gas space of a liquid-helium Dewar, so that a wide range of operating temperatures could be readily achieved. Temperatures were recorded with a calibrated Si diode mounted on the chip holder. With a temperature in the range $20 \mathrm{~K}-60 \mathrm{~K}$, a dc current $\mathrm{Ij}$ is applied until the critical current has been reached, typically $200 \mu \mathrm{a}$ to $800 \mu \mathrm{a}$ for 2 junctions in parallel.

The onset of heterodyne oscillation in the range $0.2-2 \mathrm{GHz}$ can then be observed during fine adjustment of $\mathrm{Ij}$, with the aid of 


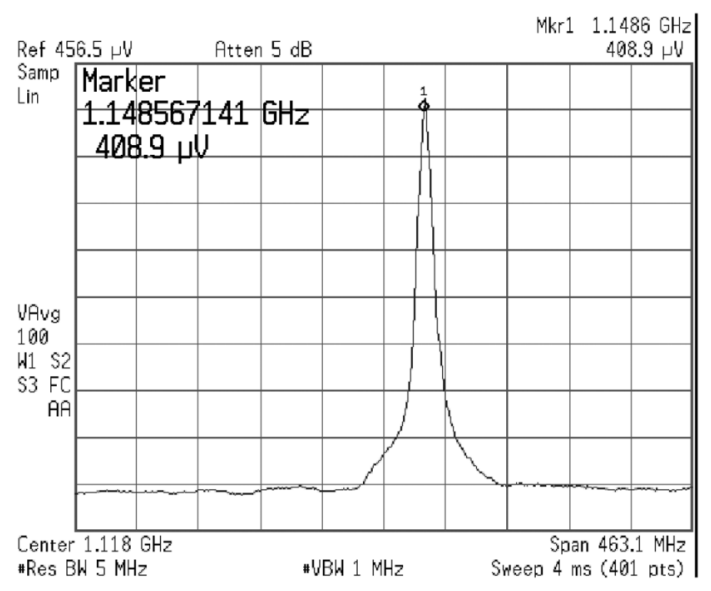

Fig. 6. Spectrum analyser trace obtained by suitable adjustment of the bias current Ij. A low-noise broadband rf pre-amplifier (gain $20 \mathrm{~dB}$ ) was used.

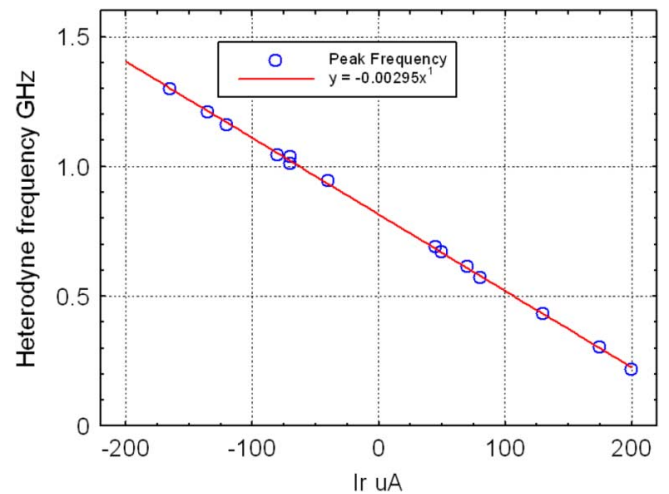

Fig. 7. Dependence of heterodyne frequency on current Ir applied to the resistor $R_{\mathrm{s}}$.at $\mathrm{T}=27 \mathrm{~K}$. Negative slope is due to arbitrary choice of polarity of current connections.

a spectrum analyser. When a peak is located, (see Fig. 6) its amplitude is optimized by fine adjustment of Ij. Then by applying current $I_{r}$ to the resistor $R_{s}$, the frequency can be adjusted. The range of frequency explored here, but by no means set by these limits, is $0.2-1.5 \mathrm{GHz}$, as illustrated in Fig. 7. The amplitude of the heterodyne oscillation depends on the optimum magnitude of the bias current, which is closely related to the junction critical current, which in turn is temperature dependent. The close correspondence between the temperature dependence of the junction bias current and that of the oscillation amplitude is demonstrated in Fig. 8. The exact value of $R_{\mathrm{S}}$ is given by the slope $\Delta \mathrm{f} / \Delta \mathrm{I}_{\mathrm{r}}$. of the line in Fig. 7, and in this case was found to be $6.095 \mathrm{mohms}$ at $27 \mathrm{~K}$, confirming that the mask design for the resistors was soundly based on preliminary measurements (Fig. 3) of the specific resistance of the unstructured Au films. The upper limit on heterodyne frequency is a subject of on-going work, but is expected to be at least $10 \mathrm{GHz}$. It will ultimately be limited by parasitic effects, e.g., inductance of interconnects etc.

\section{A. Heterodyne Oscillation Linewidth}

The linewidth is fundamentally determined by Johnson noise in $\mathrm{R}_{\mathrm{s}}$, according to (1). For example, thermally-induced voltage noise in a $6 \mathrm{mohm}$ resistance corresponds to a linewidth of order

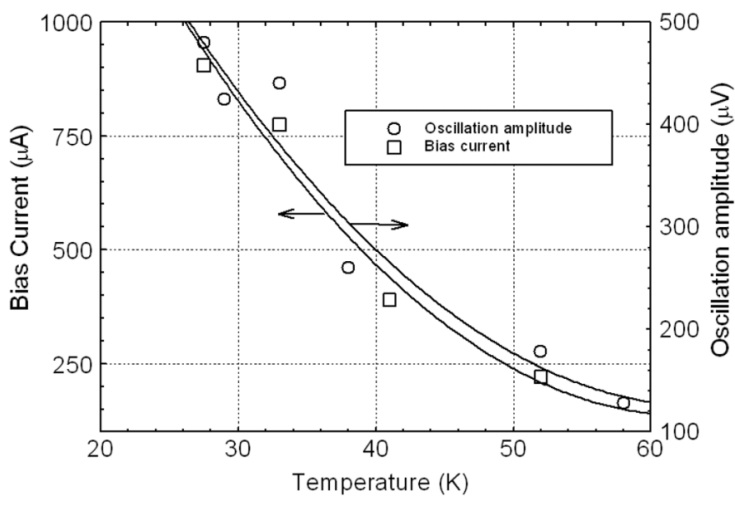

Fig. 8. The junction optimum bias currents (open squares, left axis) and the heterodyne oscillation amplitudes (open circles, right axis) have similar temperature dependences. The solid lines are best quadratic fits to the two data sets.

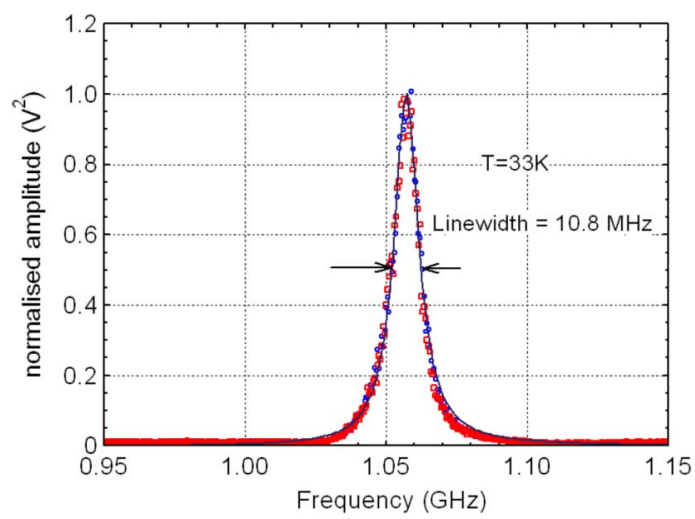

Fig. 9. Example of heterodyne oscillation linewidth measurement. The oscillation amplitude was squared and peak normalized to 1 .

$8 \mathrm{MHz}$ at $33 \mathrm{~K}$, whereas linewidths observed in our present work are around $10 \mathrm{MHz}$, Fig. 9. A possible cause of excess linewidth broadening is the fact that HTS junctions universally have been shown to have excess voltage noise due to criticalcurrent fluctuations [8], which translates to frequency "jitter" via the ac Josephson effect. This source of noise is generally ascribed to the trapping-de-trapping of Cooper pairs at sites within the grain-boundary junction, and as such, is largely independent of the fabrication technique. It is noteworthy that R-SQUIDs made from low-temperature-superconducting thin films, e.g. Niobium, in which the tunnel barrier is relatively free of such defects, show greatly reduced, even negligible, linewidth broadening, and for this reason, have been serious contenders for the realization of an absolute noise thermometer [9].

Another possible source of additional linewidth broadening may be external magnetic field noise or current noise in bias supplies, both of which can phase-modulate the heterodyne signal [10]. A frequency dependence of the linewidth is not expected, because according to (1), the ratio $\Delta \mathrm{f} / \mathrm{T}$ should be independent of frequency and of temperature, for a given value of $R_{S}$. Nevertheless, over the frequency range covered in this paper $(0.2$ $\mathrm{GHz}-1.5 \mathrm{GHz}$ ), and temperatures between $27 \mathrm{~K}-58 \mathrm{~K}$, a weak frequency dependence was observed, as shown in Fig. 10. This may be explained by the fact that we have not allowed for a possible frequency- or temperature-dependence of $R_{s}$. A more detailed evaluation of the noise temperature and linewidth broad- 


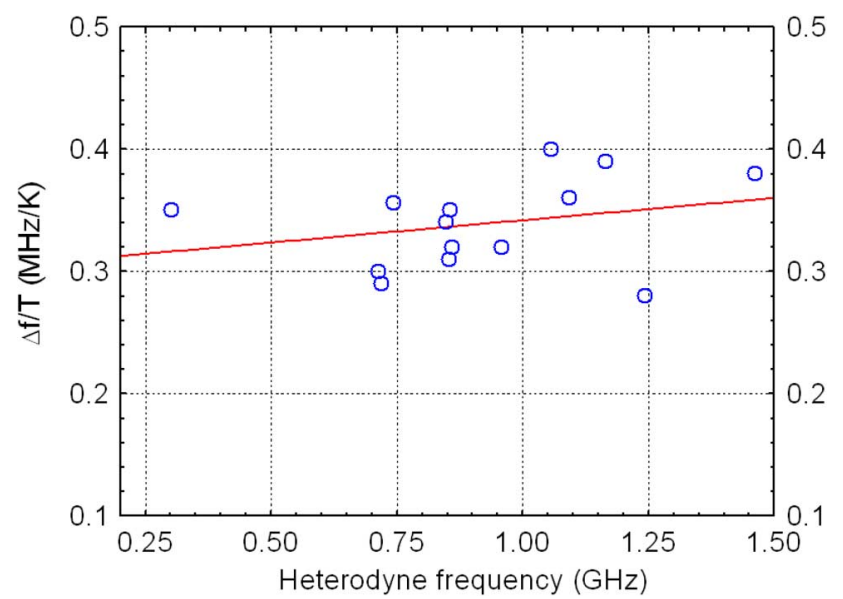

Fig. 10. The linewidth/temperature ratio showed a weak dependence on the heterodyne frequency.

ening in HTS R-SQUIDs has been recently published [12], and following on from that work, a thorough investigation of all linewidth broadening mechanisms will be carried out.

It is interesting at this point to refer to an alternative type of superconducting Josephson-effect oscillator which has been thoroughly investigated in recent years: the Josephson FluxFlow Oscillator (FFO) [11]. Operating at frequencies of 500 $\mathrm{GHz}-700 \mathrm{GHz}$, free-running linewidths around $10 \mathrm{MHz}$ have been reported. Additional sources of noise were partly attributed to resonances or other spurious effects associated with the relatively complicated tuning arrangement, which required the application of a variable magnetic field. Those helium-temperature FFO devices have not however, to our knowledge, been realized in HTS materials.

\section{CONCLUSION}

The design, modeling, fabrication and experimental demonstration of Josephson heterodyne oscillators providing useful rf outputs at frequencies up to $1.5 \mathrm{GHz}$ and temperatures up to 58 $\mathrm{K}$ have been carried out. Predictions of JSIM models have been verified, and new directions for further research, primarily into the causes of linewidth broadening, have been indicated.

\section{ACKNOWLEDGMENT}

The authors thank colleagues at CSIRO (Australia), NPL (UK) and to former students at Strathclyde University (UK), for valuable contributions. JCM thanks CSIRO for technical and scientific input, and for hospitality during several visits.

\section{REFERENCES}

[1] G. S. Krivoy and H. Koch, "An all thin-film resistive DC SQUID-A current-controlled oscillator," Supercond. Sci. Technol., vol. 5, p. 605 , 1992.

[2] C. M. Pegrum and J. C. Macfarlane, "Self-induced structure in the current-voltage characteristics of RSQUIDs," presented at the ASC08 , , unpublished.

[3] J. C. Macfarlane, L. Hao, D. A. Peden, and J. C. Gallop, "Linewidth of a resistively shunted high-temperature-superconductor Josephson heterodyne oscillator," Appl. Phys. Lett., vol. 76, pp. 1752-1754, 2000.

[4] J. Du, S. K. H. Lam, and D. L. Tilbrook, "Metallization and interconnection of HTS YBCO thin film devices and circuits," Supercond. Sci. \& Technol, vol. 14, pp. 820-825, 2001.

[5] C. P. Foley, E. E. Mitchell, S. K. L. Lam, B. Sankrithyan, Y. M. Wilson, D. L. Tilbrook, and S. J. Morris, "Fabrication and characterisation of YBCO single grain boundary stepedge junctions," IEEE Trans. Appl. Supercond., vol. 9, p. 4281, 1999.

[6] J. Du, S. Gnanarajan, and A. Bendavid, Physica C, vol. 400, p. 143, 2004.

[7] Theva GmbH, Dünnschichttechnik GmbH, 85737. Ismaning, Germany.

[8] L. Hao, J. C. Macfarlane, and C. M. Pegrum, "Excess noise in YBCO junctions," Supercond. Sci. Technol., vol. 9, p. 678, 1996.

[9] R. J. Soulen, W. E. Fogle, and J. H. Colwell, "Measurements of absolute temperature below $0.75 \mathrm{~K}$ using a Josephson-junction noise thermometer," J. Low Temp. Phys., vol. 94, pp. 385-487, 1994.

[10] C. M. Pegrum, "The dynamics of high-frequency DC RSQUID oscillators," Supercond. Sci. Technol., submitted for publication.

[11] V. P. Koshelets et al., "Externally phase-locked flux flow oscillator for sub-mm integrated receivers: Achievements and limitations," IEEE Trans. Appl. Supercond., vol. 13, p. 1035, June 2003.

[12] J. Du, J. C. Macfarlane, and L. Hao, "Noise temperature and linewidth of HTS R-SQUID GHz oscillators," Appl. Phys. Lett., vol. 93, p. 033507, July 22, 2008. 